\title{
Pobreza urbana, segregación residencial y mejoramiento del espacio público en la Ciudad de México
}

ARTURO MIER Y TEBŔN", ISABEL VÁZQUEZ", ALICIA ZICEARDI

\section{Resumen}

En la ciudad de México, al igual que en las metrópolis modernas de América Latina, grandes mayorías de la población no logran acceder a los ingresos, los recursos y las oportunidades que garanticen condiciones de trabajo y de vida dignas. La pobreza, la desigualdad y diferentes formas de exclusión social afectan la cohesión social. Los diferentes métodos para estimar la magnitud y características de la pobreza permiten conocer la escala y características que adquiere esta problemática. De esta forma entre los bienes y servicios que ofrece la ciudad a quienes en ella habitan se analizan las acciones públicas emprendidas para mejorar o construir nuevos espacios públicos, para promover prácticas colectivas deportivas, culturales y recreativas - que contribuyan a fortalecer la identidad local y la vida comunitaria. Interesa recuperar particularmente la experiencia del Programa Comunitario de Mejoramiento Barrial, impulsado por el gobierno del Distrito Federal desde el año 2007, el cual constituye una masiva e innovadora acción participativa que ha logrado transformar un amplio número de espacios públicos en barrios colonias y pueblos que se encuentran en condiciones de marginación urbana y degradación social. En este artículo se analizan dos casos exitosos de transformación de espacios públicos con recursos del Programa lo cual

\footnotetext{
* Professor, Urbanista e Arquiteto - Departamento de Arquitetura da Universidad Iberoamericana Ciudad de México (México). E-mail: arturo.mieryteran@uia.mx

** Centro de Enseñanza para Extranjeros (CEPE) Universidad Nacional Autónoma de México (UNAM) (México).E-mail: mivazquez@cepe.unam.mx

*** Programa Universitario de Estudios sobre la Ciudad (PUEC) Universidad Nacional Autónoma de México. (UNAM) (México). E-mail: ziccardi@puec.unam.mx
} 
ha contribuido a la promoción de la participación ciudadana. La metodología utilizada en este trabajo es cualitativa y se sustenta en la recopilación y sistematización de fuentes documentales, entrevistas a actores institucionales y sociales y la observación participante de los autores en los casos de estudio seleccionados.

Palabras claves: Pobreza urbana. Espacio público. Derechos ciudadanos. Políticas sociales.

\section{Abstract}

In Mexico City, as well as in other Latin American modern metropolises, huge majorities among population do not get access to income, resources and opportunities that may guarantee adequate conditions living and labor conditions. Poverty, inequality and distinct ways of social exclusion affect social cohesion. The distinct methods used to assess the extent and characteristics of poverty allow for understanding the range and features assumed by this problem. Therefore, among the goods and services offered by the city to those who live in it, this study analyses the public actions taken with the purpose of improving or building new public spaces aimed at promoting collective practices - sports, cultural and recreational areas - which may contribute to strengthen both local identity and community life. It is aimed, particularly, at retrieving the experience of Programa Comunitario de Mejoramiento Barrial (Community Program for Neighborhood Improvement), an initiative of the Federal District government, in force since 2007, that constitutes a massive and innovative participatory action which succeeded in transforming a large number of public spaces in neighborhoods, districts and shantytowns that were under conditions of urban marginalization and social degradation. This article presents two successful cases, in which the governmental support program led to changes in public spaces that contributed for promoting community participation.

Keywords: Urban poverty. Public space. Citizen rights. Social policies. 


\section{Introducción}

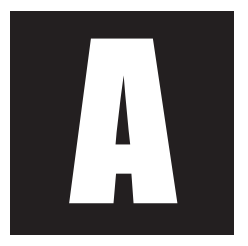

cceder a espacios públicos de calidad para realizar actividades colectivas (deportivas, culturales, sociales) favorece la convivencia ciudadana, el fortalecimiento de la identidad local y la cohesión social. ${ }^{1}$ Por ello, en contextos urbanos en los que prevalecen condiciones de pobreza las acciones gubernamentales del ámbito local se orientan actualmente no sólo a modificar las condiciones materiales de los espacios públicos degradados sino a favorecer la organización de la vida comunitaria. Así, la principal idea que organiza el análisis realizado en este trabajo es que los procesos participativos de realización material de obras en barrios populares no sólo permiten crear o mejorar espacios públicos sino que generan nuevas formas de organización social que se inscriben en los enunciados y prácticas que promueven las políticas sociales urbanas basadas en el reconocimiento pleno de los derechos ciudadanos.

En este sentido, por los nuevos procedimientos institucionales y espacios de participación que abre esta innovadora acción pública local que inaugura el Programa Comunitario de Mejoramiento Barrial (PCMB) de la Secretaria de Desarrollo Social del Gobierno del Distrito Federal (20062011) permite conocer los alcances y dificultades que enfrenta esta masiva experiencia participativa, a través de la cual se han creado o transformado un amplio número de espacios públicos en colonias, barrios y pueblos de la ciudad capital. Por ello, en primer lugar se exponen las condiciones de pobreza en la que viven grandes mayorías en barrios populares de la Ciudad de México. En segundo lugar se analizan las principales características

1 Entre otros, véase Borja (2003); Ramírez Kuri (2008); Segovia (ed) (2007). 
institucionales del PCMB y se exponen dos proyectos realizados en este programa que han permitido alcanzar importantes resultados materiales en la construcción de espacios públicos aún cuando son muy diferentes entre sí, tanto en términos de los actores involucrados como de los procesos que subyacen para alcanzar una mejor convivencia ciudadana y una mayor cohesión social. Finalmente, en las conclusiones se sistematizan un conjunto de ideas conceptuales sobre el papel del espacio público en los procesos de hacer efectivos los derechos ciudadanos.

\section{Pobreza Urbana, desigualdad social y segregación residencial}

La perspectiva desarrollada por Townsend (1979) sobre la pobreza urbana como pobreza relativa, la cual ha sido revisada en otros trabajos en función de las particularidades que adquiere la pobreza en las ciudades latinoamericanas (Ziccardi, 2006, 2008 y 2009b), apunta a describir no sólo la privación de ingresos sino de recursos en la que viven los sectores populares. Esto remite a las dificultades que deben enfrentar grandes mayorías para acceder a los bienes y servicios públicos, particularmente las que viven en las masivas y cada vez más distantes periferias de nuestras ciudades.

Lo importante es que más que en la distribución de los ingresos el énfasis se pone en la distribución de los recursos, otorgando particular importancia al hecho de que los individuos necesitan participar con patrones o trayectorias de vida de acuerdo a las costumbres y a las actividades que son propias de la sociedad a la que pertenecen. La pobreza urbana no sólo hace referencia a la capacidad de pago del trabajador y su familia para acceder a bienes en el mercado sino al nivel de vida y a la oferta diferenciada de bienes públicos que ofrece la sociedad local.

A partir de ello es posible afirmar que en las ciudades de la región “...en el espacio urbano y social es más difícil que prevalezcan situaciones 
generalizadas de pobreza absoluta, si por tal se entiende carencia de alimentación y servicios básicos. La ciudad es precisamente una aglomeración de población y de actividades que ofrece un conjunto de bienes y servicios colectivos — educación, salud, recreación, acceso a la cultura— al conjunto de la ciudadanía, independientemente de su capacidad de apropiación en el mercado" (Ziccardi, 2006). Por ello la pobreza urbana está más directamente vinculada a la dificultad de acceder a suelo barato donde producir una vivienda digna, a infraestructura básica (agua y drenaje) y a equipamiento comunitario como son: los centros de salud, las guarderías, los kinders, las escuelas y los espacios deportivos y culturales. Asimismo, hay que considerar que los obstáculos al acceso de estos bienes y servicios colectivos signan las prácticas y comportamientos sociales y culturales de los sectores populares de la ciudad y ha sido el sustento de las prácticas clientelares que han prevalecido históricamente en la relación gobierno-ciudadanía. Pero además la pobreza urbana está estrechamente vinculada a las condiciones de desigualdad socioeconómica y urbana un concepto relacional éste último que permite ubicar a individuos, colectivos sociales, barrios o zonas de una ciudad en la escala de distribución de bienes, recursos y servicios en que se localizan en el territorio.

En el caso de las ciudades mexicanas Anzaldo y Barrón (2009) afirman que en México a lo largo del siglo pasado “...mientras la población total multiplicó siete veces su tamaño, la población urbana lo hizo 44 veces, en tanto que el número de ciudades se incrementó 11 veces, lo que da cuenta del acelerado proceso de urbanización y del aumento notable en el tamaño medio de las zonas urbanas, donde a su vez se destaca la elevada concentración de personas en las principales metrópolis del país". Pero este proceso no sólo generó el pasaje de una sociedad predominantemente rural a una urbana, sino a una sociedad predominantemente metropolitana ya que actualmente más de la mitad de los mexi- 
canos viven en zonas metropolitanas. El principal rasgo de este proceso fue una expansión urbana de baja densidad, que en las últimas décadas, protagonizaron no sólo las clases populares tras la búsqueda de opciones habitacionales de bajo costo, sino también los fraccionamientos de las clases altas que viven en los llamados "barrios cerrados" en busca de protección, exclusividad y contacto con el medio ambiente natural.

De esta forma se ha identificado un proceso de urbanización de la pobreza en algunos países latinoamericanos caracterizado porque el peso de la población pobre urbana tiende a pesar ser cada vez más en el total de pobres porque la población urbana crece sostenidamente y lo hace viviendo de manera precaria, reproduciendo y el patrón de segregación residencial popular periférico.

Existe una marcada desigualdad entre los diferentes estratos sociales de la población y el territorio en que habitan en la ciudad (Veiga, 2007). Los procesos de segregación urbana muestran los límites territoriales y/o imaginarios que existen entre los espacios residenciales de los diferentes grupos sociales. En este sentido, no hay una homogeneidad estricta, o a una ausencia total de relación entre las zonas, sino que el sentido real de la segregación plantea la existencia de un dilema permanente entre las fuerzas de afinidad y los mecanismos de exclusión que pueden promoverla, lo cual parece ser una situación característica de la vida de las ciudades (Arriagada y Rodríguez 2003). Así, se configuran territorios segregados que son indicadores de la "nueva pobreza urbana" y la creciente debilidad de los vínculos de los pobres con el mercado de trabajo, así como también su aislamiento progresivo con respecto a otras clases sociales (Kaztman, 2003).

Por ello, en un contexto de creciente desigualdad y exclusión social para grandes mayorías los espacios públicos juegan un papel de primordial importancia ya que cumplen importantes funciones sociales y urba- 
nas al contrarrestar estos procesos y contribuir a avanzar en el ejercicio del derecho a la ciudad como un derecho de todos los ciudadanos (Cfr. Carta del Derecho a la Ciudad de México). Lo público urbano se materializa en estos espacios que son un lugar de encuentro y de convivencia ciudadana, en los que se desarrollan diferentes actividades colectivas y comunitarias que logran poner freno a la fuerte individuación social, al favorecer la aparición y/ o fortalecimiento de una identidad colectiva (Ramírez Kuri, 2008; Segovia (ed), 2007).

En este sentido, las llamadas políticas urbanas de inclusión social

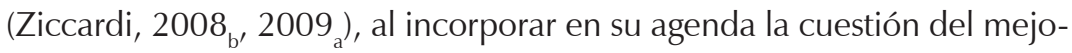
ramiento o creación de espacios públicos, no sólo plantean la realización de obras materiales sino generar condiciones para avanzar en los procesos de ampliación de los derechos sociales, culturales y ambientales para quienes viven y trabajan en la ciudad. De esta forma, la construcción y uso colectivo del espacio público promueve la participación de la ciudadanía y permite avanzar en el ejercicio pleno del derecho a la ciudad.

En la ciudad de México, una de las megalópolis del mundo, los pueblos, colonias y barrios populares son universos socio-económicos y urbanos en los que prevalecen condiciones de privación o precariedad de bienes y servicios básicos. Cuando se crean entornos urbanos que promueven la convivencia comunitaria y la cohesión social se pueden contrarrestar estos procesos y contribuir a mejorar sustancialmente la calidad de vida.

Debe tenerse en cuenta que la eficacia de las políticas sociales urbanas de creación o renovación de espacios públicos en las que participa activamente la ciudadanía depende de: i) la capacidad organizativa y la experiencia previa en materia de trabajo colectivo que han tenido los ciudadanos en su propio barrio; y ii) las nuevas relaciones de corresponsabilidad que entablan las instituciones gubernamentales encargadas de la política social con la ciudadanía y sus organizaciones, en un contexto de democratización 
de la gestión local. Desde la perspectiva de las políticas de inclusión social esto supone repensar el diseño e implementación de los programas sociales a partir de la experiencia desarrollada en los últimos años, considerando el valor pedagógico que encierran estos ejercicios de participación ciudadana en la construcción de una cultura democrática. En particular, lo que interesa analizar en este trabajo es la forma cómo los sectores populares logran aprender a participar en una decisión pública y obtener mejores resultados en las obras, tanto en términos de calidad constructiva y urbana, como de eficiencia financiera, cuando ellos se encargan de la administración de los recursos, como es el caso del Programa de Mejoramiento de Barrios aquí analizado. Sin duda estos procesos requieren esfuerzo, tiempo y dedicación pero contrarrestan las prácticas de exclusión de que son objeto las clases populares y permiten avanzar en el camino de disminuir las marcadas desigualdades sociales y urbanas que existen en nuestras ciudades.

\section{Las mediciones de la pobreza en el DF}

Como resultado de la migración campo-ciudad, la expansión de asentamientos irregulares, la precariedad en la vivienda y la falta de servicios, pero sobre todo por las condiciones de informalidad y bajos salarios, la pobreza en la Ciudad de México ha alcanzado cifras significativas, no sólo en los municipios de la zona metropolitana sino en la ciudad central. Las mediciones de este fenómeno arrojan diferentes resultados según las variables que se consideran y en este trabajo se expondrán cuatro metodologías y los resultados que las mismas arrojan para el caso del Distrito Federal.

- Línea de pobreza

Para medir el fenómeno de la pobreza en el Distrito Federal el Consejo Nacional de Evaluación de la Política de Desarrollo Social (CONE$\mathrm{VAL}$ ) organismo del gobierno nacional, aplicó inicialmente el método de 
las líneas de pobreza establecidas a partir del costo de la canasta normativa alimentaria (CNA)². Pero la evolución de las diferentes líneas de pobreza, para el caso del Distrito Federal, muestra un comportamiento muy similar entre 1996 y 2008. Los mayores cambios que se advierten en la línea de pobreza patrimonial ya que en el año 1996 representaba el 47.8 por ciento de la población y en el año 2008 el 32.1 por ciento. La pobreza de capacidades pasó de 20.3 por ciento en 1996 a 12.3 por ciento en 2008, es decir, un decremento cercano a 10 por ciento. La población en pobreza alimentaria tuvo el menor cambio registrando un decremento de 13.7 por ciento en 1996 a 7 por ciento en $2008^{3}$.

- Medición multidimensional de la pobreza

En las recientes mediciones realizadas por CONEVAL, en las cuales se modificó su metodología de análisis para reconocer el carácter multidimensional del fenómeno de la pobreza, se advierte que en el 2010, 52 millones de mexicanos son pobres, de los cuales 35 millones (67.3\%) viven en ciudades, lo cual representa un incremento de 2 millones pobres urbanos respecto al año 2008. Este aumento está relacionado con que el poder adquisitivo de los ingresos laborales de las familias mexicanas se vio afectado por dos aspectos principales: el aumento a los precios de los alimentos y la crisis financiera que tuvo un efecto negativo sobre el mercado laboral y en consecuencia sobre el ingreso. Con esta metodología en el Distrito Federal, el número de personas en situación de pobreza creció de 2 millones 454 mil a 2 millones 526 mil.

\footnotetext{
2 La línea denominada pobreza alimentaria, indica todas aquellas personas que no tienen el ingreso suficiente para alimentarse. La línea de pobreza que resulta de considerar los gastos en alimentación, salud, vivienda, vestido, transporte y educación y se llama línea de pobreza de patrimonio. La línea intermedia que se denomina pobreza de capacidades incluye sólo gastos en alimentos, educación y salud.

3 Las mediciones de línea de pobreza para el 2010 realizadas por CONEVAL sólo consideran el nivel nacional.
} 
- Medición integrada de la pobreza (MMIP)

El Consejo de Evaluación del Desarrollo Social del Distrito Federal (EVALUA) a partir de los datos de la Encuesta Nacional de Ingresos y Gastos de los Hogares, 2010 del INEGl señala que entre 2008 y 2010 hay una caída del ingreso promedio por hogar de $12.3 \%$ a nivel nacional y de $8.11 \%$ en el Distrito Federal, que se refleja en un incremento de 2.6 puntos en la incidencia de la pobreza a nivel nacional y de 4.0 puntos en las metrópolis (sin incluir al Distrito Federal). Según este organismo en la Ciudad de México el nivel de pobreza se mantuvo prácticamente igual entre 2008 y 2010, al caer 0.1, sin embargo, la intensidad de la misma aumento más que a nivel nacional, ya que la pobreza extrema pasó de $55.5 \%$ a $57.4 \%$, es decir, los pobres se volvieron más pobres.

\section{- Índice de pobreza urbana}

Otra propuesta metodológica para dimensionar la pobreza en la Ciudad de México y su expresión a nivel territorial, es el análisis del índice de pobreza urbana, el cual permite conocer la distribución del fenómeno y definir un patrón de segregación residencial y las diferencias entre los estratos socioeconómicos de la población. Este índice evalúa el comportamiento conjunto de un amplio número de variables agrupadas en cinco componentes: población, salud, educación, empleo y vivienda, y 30 variables a partir de los cuales se definieron los indicadores tanto a nivel de las Áreas Geo-Estadísticas Básicas Urbanas (AGEBs), como de las colonias que integran a la ciudad (Vázquez, 2010). El cálculo del Valor Índice Medio (VIM) obtenido, permite clasificar unidades semejantes en lo general y definir particularidades al interior de cada caso, evaluar empíricamente las desigualdades y fragmentaciones socio-espaciales que presenta un territorio determinado (delegación o colonia) y por tanto obtener una caracterización espacial de las áreas urbanas. ${ }^{4}$

4 Dichos modelos siempre deberán ser comprobados a partir de un detallado trabajo de campo, García de León (1989); Buzai (2003); Celemín, Zulaica (2008). 
Un ejercicio realizado con esta metodología indica que con datos del censo del 2000 (Vázquez, 2010) un total de 2.80 millones de personas vivían en condiciones de pobreza alta y muy alta, representando el 32.7 por ciento de la población. En el extremo opuesto, con niveles bajos y muy bajos, se encontraba el 35.3 por ciento de los habitantes, lo cual revela la existencia de la polarización y desigualdad que prevalecen en la capital del país. Las delegaciones de la Ciudad de México que concentraron la mayor cantidad de población en condiciones de pobreza fueron: Milpa Alta, Tláhuac y Xochimilco, además de Iztapalapa. Cabe señalar que las delegaciones Milpa Alta, Tláhuac y Xochimilco tienen una baja densidad de población y se localizan en el extremo sur del Distrito Federal, área con un perfil más rural que urbano, pero quedan definidas como urbanas a partir del número de población, más que por su actividad económica o equipamientos. La situación contraria se presenta en Iztapalapa y Gustavo A. Madero, las delegaciones más pobladas de la ciudad y donde aproximadamente la mitad de su población vive en condiciones muy precarias. El análisis a nivel de colonias indica que, en la ciudad de México, hay un total de 643 colonias que muestran condiciones de pobreza, de las cuales 191 corresponden a un índice de pobreza muy alto, 402 a alto y 50 a nivel medio. Las delegaciones que tienen un mayor número de colonias en condiciones de precariedad son: Álvaro Obregón, Iztapalapa y Gustavo A. Madero, seguidas por las que presentan condiciones de ruralidad; en el extremo opuesto están Cuauhtémoc, Coyoacán, Iztacalco y Miguel Hidalgo.

El mayor número de colonias en condiciones de máxima pobreza se ubican en las delegaciones Gustavo A. Madero, Iztapalapa, Álvaro Obregón y Xochimilco. En éstas se reconoce precariedad y deterioro en el equipamiento urbano, los servicios, las viviendas y los materiales de construcción de las mismas (Véase, Mapa $\mathrm{n}^{\circ} 1$ ). En este contexto los dos casos del Programa de Mejoramiento de Barrios que se analizan en este trabajo están localizados precisamente en la Delegación Álvaro Obregón. 
Una de las aportaciones de este análisis que privilegió la variable territorial de la pobreza, es que permite definir tanto la distribución geográfica de dicho fenómeno, como el patrón de segregación residencial. Un primer acercamiento revela una marcada diferencia entre los estratos sociales de la población con mejores condiciones económicas y aquellos que viven en situaciones menos favorables y ocupan territorios de la ciudad donde la realidad del medio físico, económico y social se entrelaza para reforzar una dinámica de desigualdad (Veiga, 2007)

Así se puede afirmar, que cualquiera sea la metodología de medición de la pobreza que se adopte en el Distrito Federal existen elevados niveles de pobreza, ya que más de un sesenta por ciento de la población soporta condiciones de vida precarias. Por otra parte, que se registran en este territorio, que es la principal y más importante ciudad del sistema urbano nacional, marcados procesos de segregación residencial periférica, expresión de las profundas desigualdades económicas y sociales que prevalecen (véase, Mapa $n^{\circ} 1$ ).

Pero además las características de la segregación residencial de la Ciudad de México responden a los procesos que experimentan las grandes ciudades. Siguiendo a Kaztman (2001) se pueden identificar los siguientes:

- Patrones históricos de integración social de la ciudad que reflejan los procesos que se han desarrollado en relación con la regulación del uso del suelo, los impuestos inmobiliarios, la decisiones de las autoridades en relación con el desarrollo de asentamientos, la dotación de infraestructura y modalidades de construcción y adquisición de vivienda.

- Crecimiento acelerado de población que responde a la modernización de la ciudad; la alta densidad urbana genera la expansión del transporte y las comunicaciones que facilitan la separación de los lugares de trabajo y de residencia, que van diferenciando los precios de la propiedad en función de la localización, donde el mercado inmobiliario juega un papel determinante. 
- Las disparidades económicas que evidencian cada vez más las desigualdades sociales y profundizan distancias físicas ente las clases, lo cual guarda una estrecha relación con el empleo.

Mapa n. 1

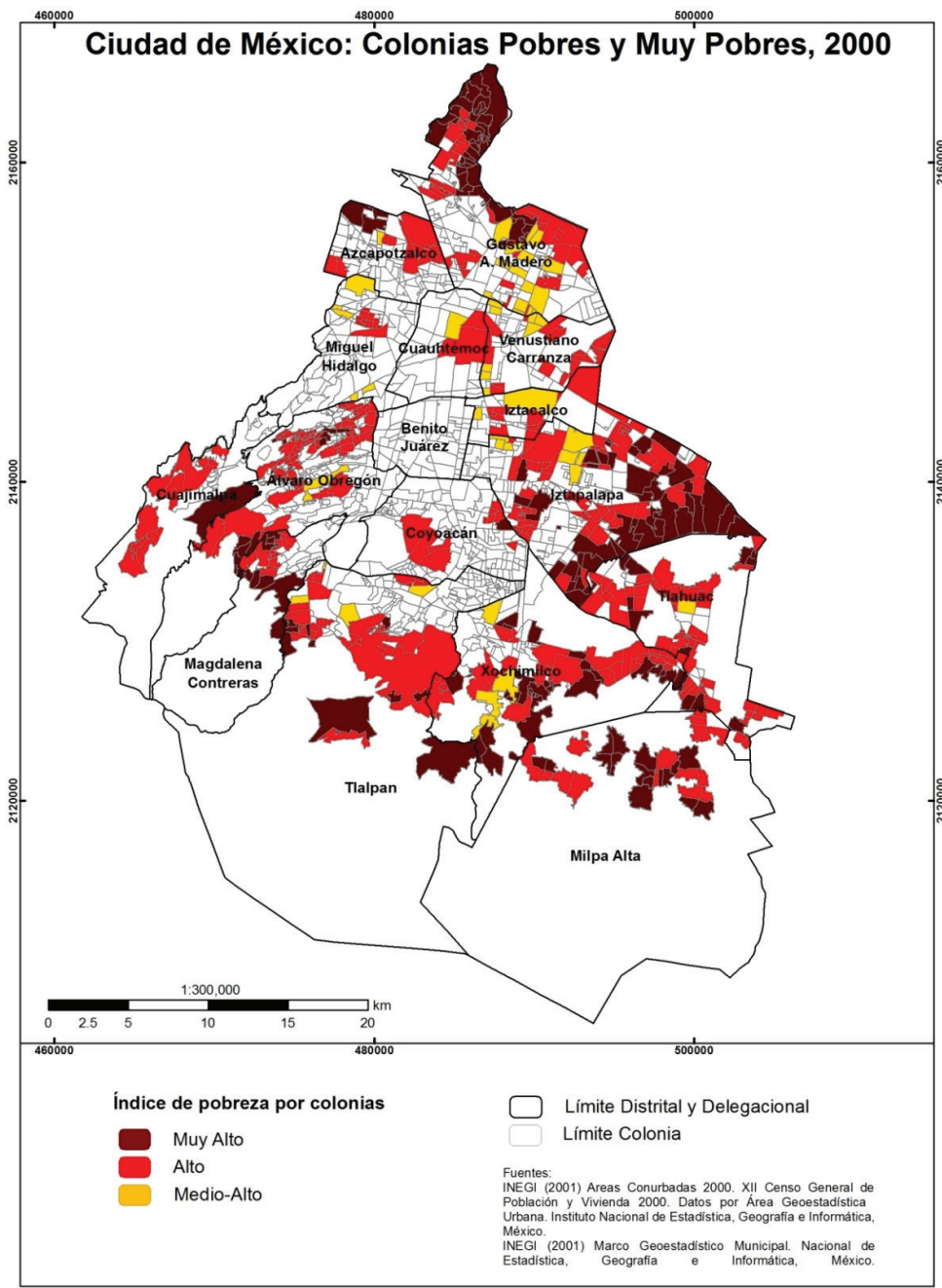


El resultado es una ciudad segmentada, fragmentada, dividida no sólo en estratos socio-económicos, entre los cuales existen profundas desigualdades en el acceso y en la calidad de los bienes de consumo básicos, servicios públicos y equipamientos urbanos. Entre éstos últimos, interesa analizar en este trabajo la cuestión del espacio público como nuevo componente de las políticas sociales y urbanas del Distrito Federal que pretende contrarrestar estos procesos de segregación residencial.

\section{El programa comunitario de mejoramiento Barrial}

A partir del año 2007 el Gobierno del D.F. asumió la necesidad de mejorar los espacios públicos de la ciudad de México como una forma de contrarrestar los elevados niveles pobreza y desigualdad que existe en su sociedad y su territorio. Como parte de una innovadora política de inclusión social se diseñó y se puso en marcha el Programa Comunitario para el Mejoramiento Barrial (PCMB) el cual convocó a organizaciones sociales, civiles, comunitarias, grupos de vecinos e instituciones académicas interesados en promover procesos participativos de mejoramiento en pueblos, barrios y colonias del Distrito Federal, particularmente en aquellos espacios que registran altos grados de conflictividad social y degradación urbana, clasificados como de media, alta y muy alta marginación (Convocatoria del Programa Comunitario de Mejoramiento Barrial, 2007, GDF). La forma de operación de este Programa se realiza de acuerdo a los criterios establecidos en las reglas de operación que anualmente se publican en el Diario Oficial del GDF y que se sintetizan seguidamente. 
Tabla 1. Principales componentes y procedimientos del PCMB

\section{Lineamientos y mecanismos de operación del Programa Comunitario de Mejoramiento Barrial}

- Procedencia de los recursos: fiscales

- Convocatoria: concurso anual

- Elegibilidad: colonias con unidades territoriales con grado de marginación medio y alto.

- Características de los proyectos: creación de espacio público e infraestructura social

- Recepción y selección de proyectos realizados por el Comité Técnico Mixto

- Monto de apoyo por proyecto comunitario: promedio un millón de pesos por tres años entre 2007 y 2009, disminución del monto en función del número de proyectos y los recursos disponibles a partir de 2010.

- Promoción: ciudadanos y organizaciones comunitarias

- Diseño: nuevas formas de participación ciudadana

- Ejecución de los proyectos: autoproducción o licitación pública

- Administración y supervisión: comités vecinales electos en asamblea comunitaria

- Asesoría técnica: arquitectos, organizaciones sociales y civiles y universidades.

- Asignación y comprobación de recursos: informe mensual.

- Evaluación de resultados: informe anual y taller general de evaluación

- Controversias: conciliación Secretaría de Desarrollo Social, Procuraduría Social del GDF 
El Programa publica en periódicos de circulación nacional una convocatoria anual y a partir de la misma, las colonias más pobres de la ciudad pueden presentar proyectos al concurso, los cuales deben ser avalados en una asamblea comunitaria ${ }^{5}$. Los proyectos seleccionados por el Comité Técnico y reciben un apoyo económico anual, el cual puede renovarse hasta por tres años consecutivos.

El Comité Técnico Mixto del PCMB está constituido por funcionarios del GDF, representantes de las Organizaciones Sociales y Civiles (OSC) y académicos, el mismo está encargado de revisar y valorar los proyectos con base en los lineamientos contenidos en las reglas de operación y seleccionar los que recibirán recursos. Estos resultados son dados a conocer en los periódicos de circulación nacional.

El principal componente es que el PCMB se sustenta en nuevas formas de participación de la ciudadanía, en particular la realización de asambleas vecinales donde se aprueba el proyecto de construcción de nuevo espacio público o renovación del existente. Este proyecto debe ser realizado con asesoría técnica y social, y presentado por un promotor o promotora vecinal. En caso de ser seleccionado se elige en asamblea comunitaria un comité de administración y otro de supervisión, constituido cada uno por tres miembros del barrio, colonia o pueblo.

Otra de las características principales del PCMB es que no se refiere a una iniciativa gubernamental sino a un proyecto que surge de la capacidad de conjuntar las ideas y experiencias que sobre este tipo de acción colectiva poseían ya algunos funcionarios locales, miembros de OSC y académicos.

5 El grado de marginación es una clasificación elaborada por el Consejo Nacional de Población de México (CONAPO) y que para fines operativos la Secretaria de Desarrollo Social del GDF la adopta para clasificar las colonias que pretenden participan en Programa. 
La idea fuerza de este Programa es reconocer que la construcción de la ciudad, especialmente en la periferia de la Ciudad de México, durante más de tres décadas, fue producto del trabajo de la ciudadanía y sus organizaciones populares que crearon masivos barrios, actualmente consolidados gracias al esfuerzo y la capacidad de organización de sus habitantes.

La delimitación del barrio o pueblo que puede concursar es el que la comunidad reconoce como tal y sobre la cual se conforma su identidad. Es decir, más allá de límites administrativos existe entre la ciudadanía un sentido de pertenencia a determinado espacio social lo cual se traduce entre otras cosas en prácticas solidarias compartidas como las que se desarrollan en este Programa las cuales contribuyen a contrarrestar los fuertes procesos de segmentación social e individualización que se advierten en nuestras ciudades. La principal fuente de financiamiento del PCMB proviene del presupuesto del GDF, a lo que se suman las obras o aportaciones que realizan las delegaciones e inclusive otras fuentes de financiamiento público y privado. Un máximo del cinco por ciento del monto recibido se destina al pago de la asesoría técnica, realizada por profesionistas provenientes de diferentes ámbitos de la sociedad civil (principalmente OSC y universidades). 
Sociologias, Porto Alegre, ano 14, no 30, mai./ago. 2012, p. 118-155

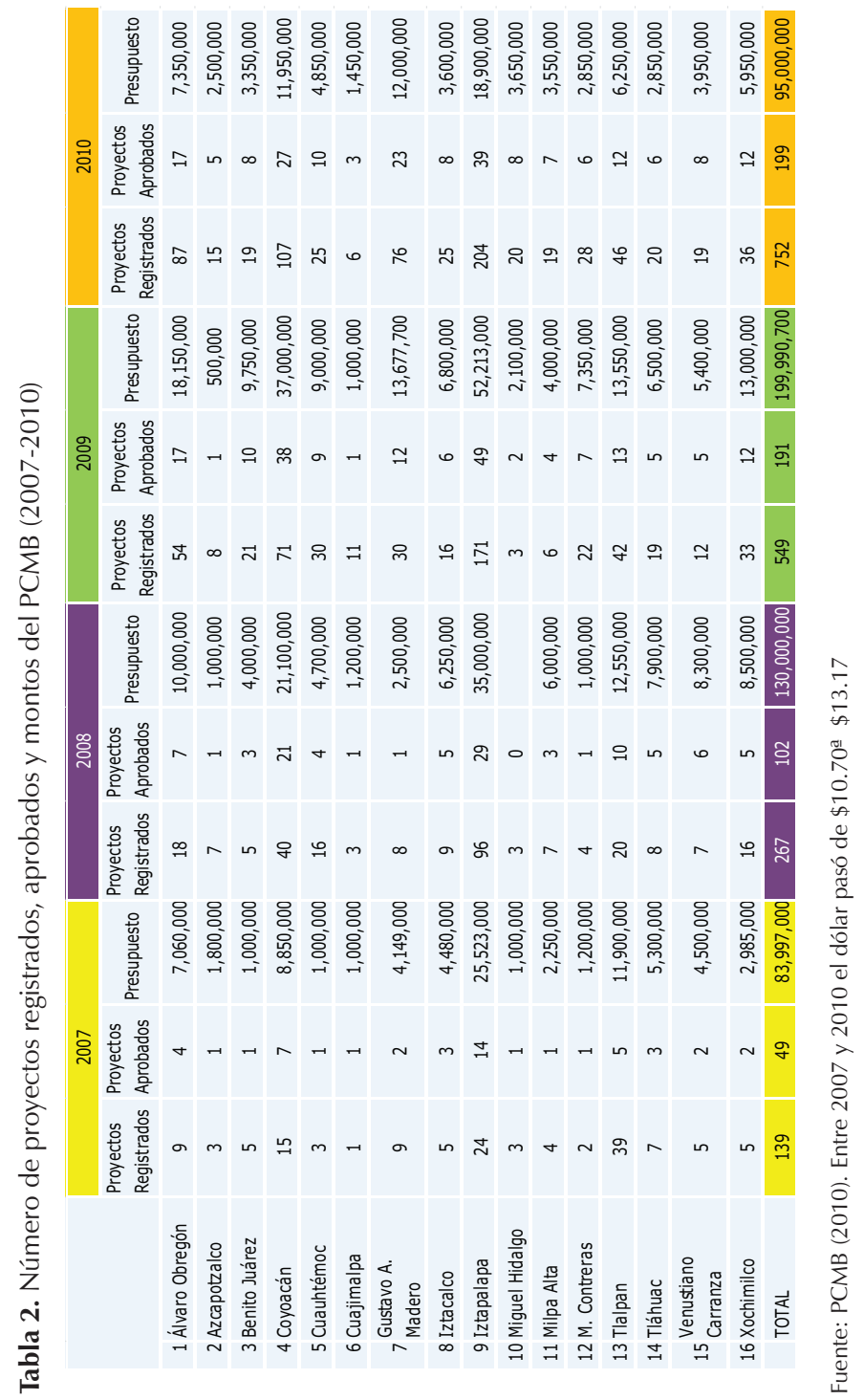


En los primeros años del programa (2007-2009) cada proyecto recibió un financiamiento de un millón de pesos (alrededor de 93,000 mil dólares) a cinco millones de pesos (alrededor de 460 mil dólares) por tres años como máximo. En los años siguientes, a pesar de los reconocimientos que recibió tanto del ámbito nacional como internacional, por ser una propuesta innovadora y participativa, la Asamblea Legislativa del Distrito Federal aprobó un monto menor de recursos para este programa. Por ello, al incrementarse el número de propuestas, el Comité Técnico tuvo que disminuir drásticamente el monto de los recursos otorgados a cada proyecto, siendo para el 2010 de 500 mil pesos (alrededor de 37 mil dólares)-

En este trabajo se han seleccionado dos proyectos localizados en la Delegación Álvaro Obregón ubicados en colonias con población de escasos recursos: proyectos exitosos aunque poseen características diferentes en cuanto a los procesos de realización del proyecto y las formas de participación de los miembros de la comunidad.

\section{Dos casos del PCMB en la Delegación Álvaro Obregón.}

Los dos casos del PCMB seleccionados para analizar el papel que puede jugar los procesos de construcción o mejoramiento de espacio público en el fortalecimiento de la identidad local y la vida comunitaria se localizan en la delegación Álvaro Obregón (ver Mapa n¹). Estos casos son: Conciencia Proletaria y el Centro Cultural y Deportivo La Hormiga del pueblo de Tizapán, los cuales se analizarán seguidamente desde una perspectiva histórica, organizativa y urbana. Sin embargo, a fin de que puedan advertirse más claramente las similitudes y diferencias se elaboró la siguiente tabla $n^{\circ} 3$, en la cual se condensan las principales características de cada barrio. 
Tabla 3. Estudio de caso, características económicas y sociales

\begin{tabular}{|c|c|c|c|}
\hline & & $\begin{array}{c}\text { Colonia Conciencia } \\
\text { Proletaria }\end{array}$ & $\begin{array}{l}\text { Pueblo de } \\
\text { Tizapán }\end{array}$ \\
\hline & Localización & $\begin{array}{l}\text { 1a Cerrada de } 16 \text { de } \\
\text { Septiembre S/N, entre } 11 \text { de } \\
\text { noviembre y } 16 \text { de } \\
\text { Septiembre }\end{array}$ & $\begin{array}{l}\text { Fraternidad No. 2, Barrio la } \\
\text { Otra Banda, San Ángel }\end{array}$ \\
\hline & Delegación & Álvaro Obregón & Álvaro Obregón \\
\hline & Tipo de obra & Centro Social & $\begin{array}{l}\text { Centro cultural y deportivo } \\
\text { "La Hormiga" }\end{array}$ \\
\hline & $\begin{array}{l}\text { Montos recibidos } \\
\text { (pesos) }\end{array}$ & $\begin{array}{l}\text { 2007: } 1 \text { mill. 2008: } 1 \text { mill. } \\
\text { 2009: } 350 \text { mil }\end{array}$ & $\begin{array}{l}\text { 2007: } 1.5 \text { mill. 2008: } 2.5 \text { mill. } \\
\text { 2009: } 700 \text { mil }\end{array}$ \\
\hline & Organización social & $\begin{array}{l}\text { Asociación Civil: Conciencia } \\
\text { Proletaria }\end{array}$ & Comunidad de Tizapán \\
\hline & Organización previa & ALTA & ALTA \\
\hline Nivel de: & Participación vecinal & Consolidada y representativa & $\begin{array}{l}\text { Liderazgo fuerte y bajo } \\
\text { consenso }\end{array}$ \\
\hline $\begin{array}{l}\text { Composición } \\
\text { comité: }\end{array}$ & $\begin{array}{l}\text { Administración } \\
\text { Supervisión }\end{array}$ & $\begin{array}{l}3 \text { Hombres } \\
3 \text { Hombres }\end{array}$ & $\begin{array}{l}2 \text { Mujeres } 1 \text { Hombre } \\
2 \text { Mujeres } 1 \text { Hombre }\end{array}$ \\
\hline Relación con: & $\begin{array}{l}\text { Delegación } \\
\text { SDS }\end{array}$ & $\begin{array}{l}\text { Autónoma } \\
\text { BAJA }\end{array}$ & $\begin{array}{l}\text { Subordinada } \\
\text { ALTA }\end{array}$ \\
\hline & Otras organizaciones & PT & Plural \\
\hline & Evaluación General & POSITIVA & POSITIVA \\
\hline
\end{tabular}

Fuente: Elaboración propia.

\subsection{La construcción del Centro Social de la Colonia Conciencia Proletaria}

- Historia y caracterización de la colonia

Conciencia Proletaria es una colonia de reciente creación reconocida legalmente a partir de 2005, aunque no aparece aún en la cartografía oficial del INEGI, ni en los planos urbanos de la Ciudad de México. A partir de una imagen de satélite se puede percibir que se localiza en la parte 
central de la delegación Álvaro Obregón, limita al noreste con la barranca, al sureste con un plantel del Colegio Nacional de Educación Profesional Técnica (CONALEP), al suroeste con un conjunto habitacional de clase media, al norte y noroeste con un terreno baldío. Su acceso principal es la avenida 5 de mayo.

Según el relato de los colonos su conformación inicia cuando un pequeño grupo de pobladores invadieron un lote baldío y promovieron un proceso de participación comunitaria. La colonia surge con el apoyo de los movimientos populares urbanos y con un intenso trabajo comunitario $^{6}$. Cabe señalar que los movimientos populares en la delegación Álvaro Obregón participan en dos organizaciones: la Coordinadora del Ex-ejido San Bernabé Ocotepec y la Unión de Colonos, Inquilinos y Solicitantes de Vivienda -11 de Noviembre (UCISV): Esta última en 1996, junto con el Partido del Trabajo, lanzó una convocatoria para ocupar el lugar donde se localiza actualmente la colonia Conciencia Proletaria, a la que acudieron grupos de familias demandantes de vivienda. Esto favoreció, desde el principio, el fuerte sentido de identidad que poseen estos colonos. Para cuidar el terreno se instalaron, en puntos estratégicos, 26 familias es decir, 100 personas, bajo condiciones muy precarias, compartiendo seis baños y usando una caverna como fosa séptica; allí permanecieron hasta que se hizo la adjudicación de los lotes. Después de un año de reuniones y crear una organización, en 1997 se fundó la Asociación Civil Conciencia Proletaria y, en agosto de ese mismo año, se realizaron movilizaciones para conseguir el apoyo de la Secretaría de Desarrollo Urbano y Vivienda del Distrito Federal (SEDUVI) y poder comenzar a construir las nuevas viviendas.

$\overline{6 \text { Con la finalidad }}$ de lograr una mejor articulación de las demandas populares, a partir de movilizaciones conjuntas y fortalecer las negociaciones unificadas, en 1982 se crea la Coordinadora Nacional del Movimiento Urbano Popular (CONAMUP) y la Regional del Valle de México dividida en cuatro regiones que integran a las delegaciones y municipios del Estado de México donde el MUP tiene mayor presencia y concentra sus principales fuerzas. 
La SEDUVI estableció un acuerdo con los inquilinos solicitantes para que la asociación civil fuera beneficiaria del predio llamado: "Las Guadalupes" actualmente Conciencia Proletaria, con un área de 1.4 ha donde actualmente viven aproximadamente 500 personas, en 100 viviendas y 20 lotes que están actualmente en construcción. El requisito fue que se hicieran los trabajos técnicos necesarios para acondicionar el terreno bajo la supervisión de la Secretaría, ya que en estudios previos de mecánica de suelos, este terreno se consideraba como una zona con riesgo por haber existido minas en esa zona. Una vez que los vecinos tomaron la responsabilidad de acondicionar el terreno, la asociación civil Conciencia Proletaria estableció un reglamento y organizaron comisiones de trabajo. La comisión técnica era la encargada de la recuperación del terreno y del urbanismo y la comisión de finanzas administraba el dinero de los socios, quienes aportaban ciento ochenta pesos mensuales. Con los fondos recaudados de estas cuotas se contrató a un arquitecto, un ingeniero, un topógrafo, un operador para una máquina financiada por todos los colonos y se nombró un residente de obra que se encargó del abastecimiento de los materiales. Asimismo, las comisiones mencionadas organizaron grupos con un jefe que era el responsable de realizar faenas de trabajo para el acondicionamiento del terreno. Tras cuatro años de intensa labor comunitaria, con cuatro estudios de mecánica de suelos, en el año 2002, la SEDUVI determinó que la regeneración del suelo era apta para uso habitacional. Cuando se entregó el terreno, la Delegación Álvaro Obregón hizo un reconocimiento a la calidad del trabajo realizado por los colonos para la recuperación y estabilización de los suelos minados el cual superó lo realizado por las constructoras privadas.

En 2005, cuando se terminó de introducir los servicios urbanos se suspendió el cobro de cuotas y, con el capital sobrante, más la recuperación de pagos atrasados, se formó un fondo revolvente que se utiliza para la realización de obras en la comunidad, lo cual evita el retraso en 
su construcción por falta de recursos. Una vez que se concluye la obra, el presupuesto resultante se divide entre toda la comunidad y se repone el dinero del fondo.

Actualmente, la colonia mantiene una estructura y organización basada en el trabajo colectivo. Los jefes de grupo se reúnen los jueves para organizar las faenas futuras y se trabaja en ellas los fines de semana. Cuando no hay obra pendiente, los socios convocan a asamblea, los domingos cada quince días, para que la comisión de finanzas haga público un reporte actualizado que fortalece la confianza y transparencia en el manejo de recursos; este espacio también se aprovecha para tratar asuntos que atañen a toda la comunidad.

Una característica importante de la comunidad es que casi todos los integrantes de la familia, a excepción de los niños, desempeñan alguna actividad por la que reciben una remuneración, situación que contribuye a mejorar el ingreso familiar. La mayoría trabaja en el sector de la construcción y los servicios, en condiciones de informalidad, los bajos salarios y falta de seguridad social.

En relación con la fisonomía actual de la colonia, se pueden identificar procesos diferenciados: tanto en el desarrollo de las viviendas, como en la calidad de los materiales, lo cual indica las diferentes condiciones económicas de los ocupantes. En este sentido, se ha gestionado la venta de algunos terrenos en la colonia y con ello han Ilegado nuevas familias. Estos mecanismos mercantiles transforman el proceso de producción social de las viviendas lo cual se expresa en la fisonomía de las mismas y en valoriza las zonas en las que se localizan. Desde el punto de vista social y organizativo el gran desafío es su integración a la comunidad y por tanto evitar que se debilite la cohesión social.

El suelo de la colonia que se halla en proceso de regularización tiene un uso principalmente habitacional con la presencia de una actividad 
comercial muy incipiente constituido por pequeñas tiendas de abarrotes, una papelería y una verdulería. Los vecinos consideran que las pequeñas tiendas tienen muchas ventajas ya que están cerca de sus casas y, además suelen financiar a los consumidores el pago de los productos.

Pero la principal ventaja que tiene la colonia Conciencia Proletaria es su localización, entre avenida Centenario y Avenida 5 de Mayo, lo que facilita a los vecinos servicios de transporte y accesibilidad a las principales vías de comunicación y otorga un valor adicional al suelo de la colonia. Asimismo, el componente social, la capacidad de organización de estos ciudadanos es el principal elemento a tener en cuenta para explicar las transformaciones logradas en su espacio público con la realización del proyecto del PCMB.

- La participación de la comunidad en el PCMB

En el año de 2008 los habitantes de la colonia Conciencia Proletaria, decidieron participar en la convocatoria del PCMB, solicitando la asesoría técnica de la Universidad Iberoamericana de la Ciudad de México. El proyecto presentado fue la construcción de un Centro Social. Para ello se realizaron reuniones en la comunidad, que se caracterizaron por una gran participación de los colonos, entre los cuales elaboraron propuestas prioritarias de mejoramiento barrial y, seleccionaron aquellas que fuesen las mejores opciones para mejorar la calidad de vida. El Plan Comunitario de Mejoramiento de la colonia Conciencia Proletaria fue desarrollado en los primeros meses y de las propuestas presentadas se seleccionó el Centro Social porque consideraron que contribuirían a desarrollar nuevas formas de participación, a mejorar la calidad de vida de la colonia y a fortalecer los procesos de construcción y ampliación de la ciudadanía. Además el Centro Social fue considerado uno de los principales puntos de encuentro de la colonia, para realizar actividades colectivas y familiares entre los niños, jóvenes y adultos. Se propuso construirlo en un predio baldío, sin uso 
fijo, y en función de la normatividad de uso de suelo correspondiente se determinó el número de niveles y área libre (Cfr. Programa de Desarrollo Urbano de la Delegación Álvaro Obregón, 1997).

Una segunda propuesta fue desarrollar el corredor peatonal y deportivo para que la colonia disponga de una zona de esparcimiento que beneficie a todos los habitantes. Para este proyecto se consideró la zona del callejón de acceso que los vecinos señalaron como peligrosa y solitaria. Para los colonos realizar actividades permanentes contribuiría a crear mayores condiciones de seguridad. Una vez cumplidos los lineamientos para ingresar al Programa Comunitario de Mejoramiento Barrial de 2008, se decidió participar sólo con el Centro Social, con la intención de crear un espacio para la convivencia familiar y la construcción de capacidades tales como el manejo de computadoras. La idea es alejar a los niños y jóvenes de los riesgos sociales existentes tales como adicciones, embarazos no planeados y delincuencia integrándolos a una vida comunitaria que sea satisfactoria para ellos. El espacio material en sí mismo no garantiza este logro sin embargo, sin embargo es una condición necesaria para que los espacios de participación ciudadana puedan funcionar. En este sentido Conciencia Proletaria es una comunidad activa que trabaja incansablemente por lograr una mejora calidad de vida para el conjunto de los pobladores de la colonia.

El proyecto resultó seleccionado por el Comité Técnico Mixto de la Secretaría de Desarrollo Social del GDF, encargado de valorar cada una de las propuestas, recibió en 2008 un millón de pesos y en 2009 se inició la construcción, caracterizada por una intensa y permanente participación social, no sólo de los directamente involucrados, sino de la comunidad en su conjunto, quien ha decidido disponer de la aportación de recursos del fondo de la colonia para solventar gastos imprevistos durante la construcción. Actualmente, el edificio del Centro Social de la colonia Conciencia 
Proletaria ha sido concluido y está funcionando plenamente para los fines que fue creado. En la convocatoria 2010 del Programa Comunitario de Mejoramiento Barrial se aprobó el proyecto de la recuperación de las áreas verdes, lo cual indica la capacidad de organización de los vecinos de la colonia para impulsar el trabajo comunitario y contar con mejores espacios públicos y recreativos que permitan el desarrollo de su colonia.

La experiencia obtenida a partir del estudio de la colonia Conciencia Proletaria, permite conocer la complejidad de los procesos de segregación residencial en la Ciudad de México, así como la importancia de la organización social y del trabajo comunitario para contrarrestar el aislamiento y hacer efectivo el ejercicio de los derechos ciudadanos para superar las condiciones de desventaja socio-económica y urbana en la que se encuentran estos sectores populares. Asimismo, la instrumentación y desarrollo del PCMB ha fomentado las prácticas de integración social de los habitantes exigiendo que las y los vecinos se organicen en los Comités de Administración y Supervisión, cuyos miembros se eligen en asamblea.

\subsection{La construcción del Centro Comunitario Cultural y Deportivo: La Hormiga del Pueblo de Tizapán, San Ángel}

\section{- Historia y caracterización del Pueblo}

El pueblo de Tizapán es uno de los pueblos originarios del Distrito Federal. Inicialmente formó parte de la localidad Tananitla la cual era un suburbio del pueblo de Coyohuacan ocupado desde 1,332 por chalcas y tepanecas y más tarde sometido por los mexicas. Al borde del pedregal y flanqueado por los ríos Magdalena y Chico, la zona fue conocida como San Jacinto a la llegada de los dominicos, San Ángel a la llegada de los carmelitas y Villa Obregón a la llegada de los revolucionarios. 
En el siglo XX el trabajo agrario convivió con las actividades fabriles localizadas en ese territorio. La fábrica de Loreto fue una de las más importantes papeleras de la ciudad actualmente convertida en los años noventa en un centro comercial y recreativo para los sectores de mayores ingresos de la ciudad. A la misma se agregaban las fábricas textiles La Hormiga, La Abeja y La Alpina, fuentes de empleo de gran importancia para los habitantes de esta zona hasta la década de los años sesenta.

Para los habitantes de Tizapán el cierre de las fábricas no sólo significó la pérdida de empleos sino el paulatino debilitamiento de la vida comunitaria ya que alrededor de estas fuentes de trabajo se organizaban buena parte de las actividades culturales, deportivas, religiosas en las que participaban activamente los habitantes del pueblo. Los actuales habitantes, que son descendientes de estos trabajadores manuales, conservan en su memoria los principales rasgos de aquel modo de vida en el que el duro trabajo en las fábricas se combinaba con el deporte y la organización de fiestas religiosas. En Tizapán, cuyo significado es "en la tierra blanca", "Río de polvo blanco", vive un pueblo que a pesar de las profundas transformaciones económicas, territoriales y sociales que se han dado en este territorio, en el último cuarto siglo, logra preservar su identidad-

Se estima que más de la mitad de la población vive en el Pueblo de Tizapán desde hace más de veinte años, siendo muchos los habitantes que descienden de familias originarias. La gran mayoría de sus habitantes se identifica con el pueblo, lo cual indica que persiste un fuerte sentido de pertenencia en esta comunidad. En el pueblo original de Tizapán, incluidas las colonias Tizapán Pueblo, Ermita, Progreso Tizapán, Progreso, Barrio de Loreto y La Otra Banda, viven actualmente poco más de 18,000 habitantes, predominando las mujeres, que representan más del cincuenta por ciento del total según datos de la Dirección de Planeación del GDF (2005). En la parte central del pueblo viven 3,782 personas, si se suma Ermita y Progreso superan las 11,000 y se registra un grado medio de marginación. 
La población adulta mayor de 70 años representa alrededor del 10 por ciento de la población, siendo predominantemente mujeres, sin embargo más allá del número se observa una intensa participación de este sector de la población en la promoción de actividades comunitarias. Dado que uno de los objetivos de este proyecto es fortalecer y preservar la identidad de este Pueblo originario para heredarlo a las futuras generaciones. Se elaboró originalmente un Plan que propone el desarrollo y ampliación de actividades colectivas que garantizarán una mayor inclusión social en el Centro Cultural y Deportivo.

Más de la mitad de la población son jóvenes o niños para los cuales el Pueblo de Tizapán ofrece pocas opciones de formación y capacitación más allá de educación formal. Las limitaciones, la ausencia y el deterioro de los espacios públicos para que realicen actividades deportivas, culturales, recreativas como lo hicieron anteriores generaciones de las cuales salieron destacados personajes de la vida nacional, fue uno de los principales fundamentos de la principal propuesta de este Plan Comunitario: transformar la cancha de futbol en un centro de desarrollo comunitario. Es decir, que no sólo se desarrollen actividades deportivas sino que se propicie la participación de las y los jóvenes en charlas y cursos relacionados con su salud reproductiva, la planeación familiar, la superación de las adicciones, la cultura, etc. Estas propuestas de la comunidad se hacen efectivas en la medida en que el gobierno local y otras instituciones, como por ejemplo las universidades, están dispuestos ofrecer diferentes actividades colectivas, casi de manera gratuita en estos espacios.

En el Pueblo de Tizapán viven familias de trabajadoras y trabajadores de la industria manufacturera y los servicios cuyas remuneraciones son bajas, como lo son para el conjunto de la ciudad. Las nuevas ocupaciones que se generaron en esta zona en el comercio y los servicios, a pesar de los compromisos asumidos, no ocuparon la mano de obra local, la cual 
debió hallar una actividad remunerada en otro sitio de la ciudad con los consiguientes desplazamientos que ello implica.

- La participación del Pueblo en el PCMB

La primera etapa para concretar un proyecto cuyo beneficio abarcara a la mayor parte de la población consistió en sensibilizar a un pequeño grupo de representantes de comités vecinales acerca de la importancia de iniciar un proceso de participación ciudadana para recuperar los espacios públicos y mejorar la calidad de vida del Pueblo de Tizapán. Para poder concretar el proyecto se llevaron a cabo recorridos por la zona con vecinos, representantes delegacionales y los equipos técnico y social de las universidades: el Instituto de Investigaciones Sociales de la UNAM y la Facultad de Arquitectura de la Universidad Iberoamericana, con los cuales se logró identificar los principales problemas urbanos, sociales, culturales y ambientales que existían en esos espacios públicos. Asimismo, se organizaron talleres de análisis y discusión con representantes y vecinos para elaborar una propuesta de recuperación de la historia del pueblo. Se aplicaron entrevistas a profundidad con jóvenes y adultos y se llevó a cabo recopilación de información bibliográfica, cartográfica y estadística de la zona.

En Julio de 2007, se organizó la primera asamblea vecinal, con la participación de más de 70 vecinos para analizar y discutir la propuesta y lograr un consenso entre los vecinos de las seis colonias, comerciantes, proveedores de servicios, representantes de la delegación Álvaro Obregón, establecer prioridades y calendarizarlas anualmente para ser presentadas al concurso del Programa de Mejoramiento Barrial en el primero, segundo y tercer año.

Se hizo, la revisión, discusión y mejoramiento del proyecto, mismo que se presento en talleres por colonia, incorporando las observaciones de los vecinos, agregando nuevas propuestas de mejoramiento urbano, a fin de definir una estrategia basada en un proceso de planeación par- 
ticipativa y de gestión asociada en la que participaron la comunidad, las diferentes dependencias de gobierno del Distrito Federal, la delegación Álvaro Obregón y académicos y alumnos de la UNAM y la Universidad Iberoamericana que actúan como promotores y asesores del proyecto participativo interesados en preservar la identidad y la vida comunitaria de este pueblo originario de la ciudad.

Una vez establecidos los pasos para validar el proyecto tanto a nivel de la comunidad como de las instancias de gobierno, se entregó el proyecto ejecutivo del la construcción del Centro Comunitario Cultural y Deportivo "La Hormiga" para participar en el concurso del Programa Comunitario de Mejoramiento Barrial. El proyecto fue seleccionado por el Comité Técnico Mixto quien le otorgó en 2007 un millón de pesos y en 20082.5 millones. En asambleas vecinales se informa del inicio de los trabajos y realizar la elección de los Comités de Administración y Supervisión, tal como se establece en la convocatoria y las reglas de operación del PCMB. Asimismo, se organizan reuniones informativas semanales y una característica importante en el desarrollo de este proyecto es que en su mayoría fueron mujeres las que impulsaron el proceso de promoción y realización de la obra, dieron seguimiento al proyecto y aseguraron tanto su conclusión, como la promoción de actividades culturales y deportivas en este espacio público.

Cabe mencionar que a partir de este proyecto se recuperó un espacio social urbano degradado que era utilizado solo por un grupo que promovía football obteniendo beneficios personales. Históricamente tiene gran significado, porque formó parte de los campos deportivos de las fábricas La Hormiga y la Alpina. Allí se creó un lugar de convivencia para niños y adultos con la finalidad de realizar tanto actividades deportivas como culturales y sociales, rescatando un espacio excluyente e inseguro, que resultaba poco propicio para la práctica deportiva y la convivencia de la comunidad. Sin embargo, los conflictos que existen históricamente 
en la zona entre los diferentes grupos que habitan en este pueblo urbano central impiden consolidar una nueva organización capaz de garantizar un uso público, plural y democrático en este espacio urbano.

\subsection{Un análisis comparativo}

En el análisis comparativo de los proyectos de espacio público que se incluyeron en este trabajo y que se localizan en la delegación Álvaro Obregón se observa que ambos tienen en común el tratarse de experiencias de producción social de un espacio público que contribuyen a fortalecer la convivencia y la cohesión social. Pero mientras que la localización, la historia y la existencia previa de una asociación civil en la colonia Conciencia Proletaria favorece el desempeño de las instancias organizadoras creadas a partir de participar en el PCMB (los Comités de Administración y Supervisión), en el caso de Tizapán en cambio la persistencia de disputas entre viejos liderazgos locales vinculados a los partidos políticos no se logró superar a partir de esta experiencia. Si bien en un principio diferentes vecinas y vecinos participaron activamente en el proyecto, a medida que se fueron definiendo las responsabilidades de los Comités se fueron creando conflictos, principalmente vinculados al proceso de producción de la obra y la forma de asignación de los recursos. Las reuniones semanales realizadas no fueron suficientes para ampliar la información y el proyecto en reiteradas ocasiones intentó ser modificado para responder a intereses particulares. Esto podía haberse neutralizado si las autoridades de la Delegación Álvaro Obregón hubiesen asumido un papel más institucional que político, lo cual no ocurrió. La presencia y los esfuerzo que realizaron los investigadores de la UNAM y de la Universidad Iberoamericana para realizar un proyecto con gran valor arquitectónico y urbano y con fuerte sentido social contaron con el reconocimiento 
de pequeños grupos de vecinos pero no lograron liderar transformaciones sustanciales en los comportamientos colectivos que permitiesen avanzar en la construcción de una experiencia de democracia local.

El proyecto de Conciencia Proletaria recibió una cantidad menor de recursos lo que se justifica por la dimensión de la obra y el tipo de materiales que en ésta se utilizaron. Pero la ejecución de los dos proyectos se basó en la organización social que permitió concretar la propuesta de la comunidad y dar seguimiento a la convocatoria del PCMB. En Conciencia Proletaria la participación vecinal a lo largo de todo el proyecto fue representativa en tanto que en el Pueblo de Tizapán, a pesar de que siempre se mostró un fuerte liderazgo personal, a lo largo del desarrollo del proyecto, se dio una limitada participación del conjunto de la comunidad. Sin embargo, la composición de los comités fue más plural en términos ideológicos en el Pueblo de Tizapán y las mujeres jugaron un papel decisivo de control social en la realización de la obra, su trabajo consistió en administrar y supervisar los recursos, lo cual más que empoderarlas en relación el uso y las actividades que se desarrollan en este espacio público, las colocó en una situación de permanente cuestionamiento por quienes querían asumir el control de los mismos lo cual lograron gracias al apoyo que por razones de clientela policía les dio el gobierno delegacional. Sin embargo, la honestidad de la principal lideresa, una mujer de ochenta años con una gran experiencia de trabajo social en el pueblo fue lo que garantizó la realización de la obra y la transparencia en el manejo de los recursos. También puede decirse que en este pueblo se apostó a crear instancias en las que estuviesen representados los principales grupos sociales que pertenecen a diferentes asociaciones vecinales y políticas y si bien en un principio el trabajo comunitario se sobrepuso a las diferencias, a medida que el proyecto avanzó se fueron afirmando posiciones que representaban intereses particulares y que para imponerlos recurrieron al conflicto en los procesos de toma de decisiones. 
La relación con la Secretaría de Desarrollo Social fue a la inversa sin embargo, el apoyo de la SDS no logró neutralizar los conflictos surgidos en Tizapán alrededor de la obra y el uso del espacio público. Por ello, aunque ambas experiencias pueden considerarse positivas en términos de logros materiales en ambos casos, se demuestra que los recursos gubernamentales logran mejor sus objetivos y sus alcances cuando la comunidad actúa de manera corresponsable. Sin embargo, en términos de construcción de ciudadanía y de fortalecimiento de la organización social, los resultados fueron mucho mejores en la colonia Conciencia Proletaria donde existía previamente una sólida y representativa organización social. Por ello es importante que el PCMB considere que los recursos no sólo deben asignarse para la realización de obras materiales sino también para remunerar un trabajo social profesional capaz de promover y fortalecer la organización comunitaria allí donde es débil o inexistente. Sin duda la presencia de universitarios con formación en carreras no sólo técnicas (ingenieros, arquitectos) sino en distintos campos de las ciencias sociales (sociólogos, antropólogos, politólogos) pero sobre todo participando en equipos con un gran compromiso social es lo que garantiza obtener mejores resultados en estos proyectos.

En relación con la dimensión política quizá pueda decirse que en caso de Conciencia Proletaria se advierte una pertenencia política más homogénea que sumada a la historia de la colonia favorece el trabajo colectivo y promueve procesos de tomas de decisorios democráticos. En el caso de Tizapán, en cambio, se advierte la presencia de liderazgos clientelares que forman parte tanto del Partido de la Revolución Democrática (PRD) que es el que gobierna la Ciudad de México como también de dirigentes del Partido de la Revolución Institucional (PRI) y del partido de la derecha mexicana, el Partido Acción Nacional. Es decir, existe una cultura y prácticas clientelares compartidas a la cual se enfrentan coti- 
dianamente aquellos que pretenden avanzar en la construcción de una gestión democrática del territorio, del uso de los espacios públicos.

En síntesis puede decirse que en ambos casos los resultados fueron positivos ya que se concretó tanto el Centro Social en la Colonia Conciencia Proletaria como el Centro Cultural y Deportivo La Hormiga en el Pueblo de Tizapán con los cual estas respectivas comunidades se vieron muy beneficiadas con la construcción de estos espacios públicos que ofrecen las condiciones materiales adecuadas para el desarrollo de la vida comunitaria y el fortalecimiento de la identidad local.

\section{Algunas Conclusiones}

La pobreza urbana, las situaciones de carencia y precariedad de bienes y servicios en los barrios populares de las ciudades, van más allá de las necesidades básicas de alimentación e implican la dificultad de la población de menores recursos para responder a las pautas y exigencias que establece la sociedad urbana para participar e integrarse a ella. Este tipo de pobreza se basa principalmente en la distribución y falta de calidad de los recursos (infraestructura, vivienda, empleo, representación política) más que de los ingresos.

Programas de inclusión social como el de Mejoramiento Barrial del gobierno del Distrito Federal contribuyen a mejorar la calidad de vida de los espacios urbanos habitados por los sectores más pobres de la población, creando transformaciones materiales en los espacios públicos. Pero el fortalecimiento de la vida comunitaria y la cohesión social en los sectores populares depende de otros factores sociales, psicológicos y culturales que inciden junto con los políticos en el logro de mayor organización social.

Los pueblos, colonias y barrios populares son universos socio-económicos y territoriales muy heterogéneos en los que si bien prevalecen 
condiciones de pobreza y de exclusión social, el ideal de construir espacios públicos para promover una mejor convivencia comunitaria y la mayor cohesión social, requieren de políticas sociales urbanas integradas y complejas basadas en el reconocimiento de los derechos ciudadanos, en la capacidad organizativa de los ciudadanos y en las relaciones de corresponsabilidad que éstos entablen con las instituciones gubernamentales encargadas de la política social. Esto supone incorporar junto a esta concepción de derechos la noción de responsabilidad en las acciones sociales colectivas, a fin de garantizar la producción, el acceso y el uso de los espacios públicos en condiciones de igualdad de oportunidades para todas y todos los ciudadanos sin discriminación. En síntesis puede decirse que existen experiencias muy diversas, pero estos procesos pueden ser detonadores de nuevas formas de organización social y, sobre todo, de procesos de aprendizajes colectivos compartidos para el ejercicio pleno de los derechos ciudadanos y la corresponsabilidad entre el gobierno y la ciudadanía, siempre y cuando estos sean objetivos explícitos.

Finalmente, puede decirse que en las experiencias aquí analizadas, pero muy probablemente también en muchos otros casos que se desarrollaron en el marco del PCMB, en aquellos espacios en los que existe una sólida y democrática organización social y política previa a la realización de estos proyectos al concretarse los mismos se contribuye a fortalecer el sentido de pertenencia y la convivencia comunitaria. Pero que cuando se trata de una organización previa de tipo clientelar y débil el proyecto puede contribuir a detonar nuevos conflictos y debilitar aun más los débiles lazos comunitarios. Por ello cabe señalar que lo importante no es sólo la organización previa que exista en la comunidad sino la calidad de las prácticas organizativas. 


\section{Referencias}

ANZALDO, C.; BARRÓN, E. La transición urbana de México, 1900-2005. In: La Situación Demográfica de México 2009, Consejo Nacional de Población, CONAPO, México, 2009, pp. 53-65.

ARRIAGADA, C.; RODRÍGUEZ, J. Segregación residencial en la ciudad Latinoamericana, Revista EURE, Vol. XXIX, n. 89, Santiago de Chile, 2004, pp. 5-24.

BARNES, M. Social exclusion and life course. In: BARNES, Matt et. al. Poverty and exclusion in Europe. Cheltenham (UK): Edward Elgar, 2002.

BÁRCENAS, A. Pobreza y desigualdad en el pasado reciente. Santiago de Chile: CEPAL, 2009.

BOLTVINIK, J.; DAMIÁN, A. La pobreza en el Distrito Federal en 2004. Informe final elaborado para la Secretaría de Desarrollo Social del Distrito Federal. México: El Colegio de México A.C., 2006.

BORJA, J. La ciudad conquistada. Madrid: Alianza Editorial, 2003.

BRUGUÉ, Q.; GOMÁ, R.; SUBIRATS, J. De la pobreza a la exclusión social. Nuevos retos para las políticas públicas. In: Revista Internacional de Sociología (RIS), 3a Época, n³3, sep-dic 2002 pp. 7-45.

Comisión Económica para América Latina y el Caribe (CEPAL). Panorama Social de América Latina 2008. Santiago de Chile: CEPAL, 2008.

Comisión Económica para América Latina y el Caribe (CEPAL). Panorama Social de América Latina 2009, Santiago de Chile: CEPAL, 2009.

Consejo Nacional de Evaluación de la Política de Desarrollo Social (CONEVAL). Evolución de la Pobreza en el Distrito Federal. México: CONEVAL, 2008.

Consejo Nacional de Evaluación de la Política de Desarrollo Social (CONEVAL) (2009), Comunicado de prensa No. 006/09. Dirección de Información y Comunicación Social. México: CONEVAL, Distrito Federal a 18 de julio de 2009.

CORDERA, R.; RAMÍREZ, P.; ZICCARDI, A. Pobreza, desigualdad y exclusión social en la ciudad del siglo XXI. Instituto de Investigaciones Sociales, Seminario Universitario La Cuestión Social, UNAM. México: Siglo XXI, 2008.

DAMIÁN, A. Derechos socioeconómicos y pobreza. Una alternativa para medir la pobreza en el Distrito Federal. In: YÁÑEZ-RIZO, P; LÓPEZ, A. Pobreza, desigualdad y marginación en la ciudad de México. Secretaría de Desarrollo Social del Distrito Federal, México: Dirección General de Equidad y Desarrollo Social, 2004.

Delegación Álvaro Obregón. Programa de Desarrollo Urbano, GDF, México, 1997. 
GERMANI, G. El concepto de marginalidad. Buenos Aires: Ediciones Nueva Visión, 1973.

KAZTMAN, R. Seducidos y abandonados: el aislamiento social de los pobres urbanos. In: Revista de la CEPAL n. 75, Santiago de Chile, 2001, pp. 171-189.

KAZTMAN, R. La dimensión espacial en las políticas de superación de la pobreza urbana. Serie Medio Ambiente y Desarrollo $n^{\circ}$ 59. Santiago de Chile: CEPALECLAC, 2003.

KAZTMAN, R.; RETAMOSO, A. Segregación espacial, empleo y pobreza en Montevideo. In: Revista de la CEPAL, n 85, Santiago de Chile, 2005, pp. 131-148.

NUN, J. Marginalidad y exclusión social. México: Fondo de Cultura Económica, 2001.

RAMÍREZ, P. La fragilidad del espacio público en la ciudad segregada. In: CORDERA, Ramírez; ZICCARDI, op.cit., México, 2008, pp 117-134.

ROSANVALLON, P. La nueva cuestión social. Buenos Aires: Ediciones Manantial, 1995.

SABATINI, F.; CÁCERES, G.; CERDA, J. Segregación residencial en las principales ciudades chilenas: Tendencias de las tres últimas décadas y posibles cursos de acción. In: Revista EURE vol.27, nº.82, Santiago de Chile, 2001.

SEGOVIA, O. (ed.) Espacio público y construcción social. Hacia un ejercicio de ciudadanía Santiago de Chile: Ediciones Sur, 2007.

SEN, A. El enfoque de las capacidades y las realizaciones. Pobre, en términos relativos. In: Revista Comercio Exterior, vol. 53, no. 5, Mayo, México, 2003, pp. 413-423.

TOWNSEND, P. Poverty in the United Kingdom: A Surrey of Household Resources and Living Standards. London: Allen Lane and Penguin Books, 1979.

VÁZQUEZ, I. Dimensión territorial de la pobreza en la ciudad de México, Tesis de doctorado en Sociología, Facultad de Ciencias Políticas y Sociales, UNAM, México, 2010.

VEIGA, D. Desigualdades sociales y fragmentación urbana. In: RIBEIRO, A.C. EI rostro urbano de América Latina. Buenos Aires: CLACSO, Consejo Latinoamericano de Ciencias Sociales, 2004. pp. 51-61.

YANES, P. (coord.) El derecho a la existencia y libertad real para todos. Universidad Autónoma de la Ciudad de México - Secretaría de Desarrollo Social del gobierno del DF-ICU, Grupo Promotor del Ingreso Ciudadano. 2007. 
ZICCARDI, A. Políticas de inclusión social en las sociedades complejas. In: HURTADO, S., Justicia, políticas sociales y bienestar social. UNAM, Escuela de Trabajo Social, México, 2006, pp 275-299.

ZICCARDI, A. (comp.) Procesos de urbanización de la pobreza y nuevas formas de exclusión social. Los retos de las políticas sociales de las ciudades latinoamericanas del siglo XXI. Bogotá: Siglo del Hombre, CLACSO, 2008, 420 p.

ZICCARDI, A. Políticas de inclusión social en la ciudad de México. In: BARBA, C. (comp.) Retos para la Integración Social de los pobres en América Latina. Buenos Aires: CLACSO, 2009a, pp. 237-257.

ZICCARDI, A. Ciudades latinoamericanas: procesos de marginalidad y de exclusión social. In: CORDERA, R.; RAMÍREZ, P. y ZICCARDI, A. (coord.) Pobreza, desigualdad y exclusión social en la ciudad del siglo XXI. México: IISUNAM, Editorial Siglo XXI, Seminario Universitario "La Cuestión Social", 2009b, pp. 73-91.

ZICCARDI, A. Las ciudades y la cuestión social. Quito: FLACSO-OLACCHIINNOVAR, 2009c.

Recebido em: 05.12.2011

Aceite final: 14.03.2012 